\title{
Work in progress: proactive immunization against multiple sink holes in wireless sensor network to extend its life span
}

\begin{abstract}
The contemporary world has been significantly influenced by the development of the wireless sensor network (WSN). The demand for excellent performance became a necessity; however, the restrictions in this tiny device in terms of energy resources, memory, and computational capability created numerous difficulties. Regardless of the WSN features, energy holes due to the rapid energy depletion around the sink node occur resulting in dramatic network partitioning. Previous studies have pointed out some reactive solutions to alleviate these holes. Meanwhile, proactive and early stage countermeasures are believed to serve as extraordinary immunity against this phenomenon. This work in progress aims to immunize multiple sinks against energy holes. The core of this novel proactive energy metric is a vision for the status of relative energy deviation among hole members (deputy nodes [DN]) conveyed to distant nodes in order to assist in assessing their interconnected DN relative to the density encountered by their initialized route. Supported by this proactive metric and a wise routing decision mechanism, a topology of protected multiple sinks is proposed. Thus, this competitive approach for energy-aware traffic routing ensures efficient energy balancing among the DN of each hole, plays a significant role in future routing decision, protects entire sinks from isolation, and extends its life span.
\end{abstract}

Keyword: Energy holes; Life span; Multiple sinks; Sink isolation; Tree topology; WSN 